\title{
Stability and Free Vibration Behaviour of Laminated Composite Panels Under Thermo-mechanical Loading
}

\author{
Subrata Kumar Panda • Pankaj Vaikunthbhai Katariya
}

Published online: 22 February 2015

(C) Springer India Pvt. Ltd. 2015

\begin{abstract}
In this article, the free vibration and the buckling (mechanical and/or thermal) behaviour of laminated composite flat and curved panels are analysed numerically. Simulation model has been developed using ANSYS Parametric design language code in the ANSYS environment. The convergence behaviour of present finite element results have been checked and comprehensiveness of the model is revealed by comparing the results with those available published literature. Wide variety of numerical examples is solved for different parameters such as lamination schemes, support conditions and material properties under the thermomechanical load to highlight the applicability of the present simulation model. It is finally understood that the proposed model is capable of solving the mechanical responses (free vibration and buckling) of orthotropic materials accurately with very less computational cost under the combined action of loading.
\end{abstract}

Keywords Free vibration - Stability - Laminated flat and curved panels ·

Thermo-mechanical loading $\cdot$ Finite element analysis

\section{Introduction}

Laminated composite flat and/or curved panels are increasingly used in many engineering applications such as aerospace, automotive, sports, biomedical, agriculture and marine and health instrument as well as in the other field of high modern technology. The primary benefit of composite components that can offer reduced weight and assembly simplification. The advantages of composites associated with reducing the weight of aircraft structural elements have been the principal motivation for military aviation development. The Boeing 787 (Dreamliner), have used around $50 \%$ composite materials dropping its overall weight by $12 \%$ and added strength and lower weight allow the plane to use less fuel [1]. In order

S. K. Panda $(\bowtie) \cdot$ P. V. Katariya

National Institute of Technology Rourkela, Rourkela, Odisha, India

e-mail: call2subrat@gmail.com 
to meet the economic challenges present days, it is necessary to manufacture the composite structures on the large scale to reduce the cost and easy availability of the composite structure.

It is well known that, the thin laminated structures are prone to buckle at low temperature and/or under the influence of compression load. Buckling does not mean failure of the structure; in general it is the state of geometrical instability of the structure induced by the inplane thermal/mechanical/thermo-mechanical forces. Buckling is one of the main modes of failure of the structural components when subjected to in-plane compressive stresses caused by thermal/mechanical loads. Now a day typical composite curved and/or flat panels are used in spaceships, aircraft and automobiles that are exposed to enormous acoustic, aerodynamic and inertia excitation. Vibrations with large amplitude cause great tension and subsequent reduction of life due to fatigue. As a result, the analysis of vibration and buckling of laminated structures made of composite and/or hybrid materials become significant.

Laminated composite flat and/or curved panels are being analysed based on different classical theories and shear deformation theories [2] such as the first order shear deformation theory (FSDT), the higher-order shear deformation theory (HSDT) and the classical laminated plate theory (CLPT). Thermo-mechanical buckling and vibration behaviour of laminated composite plates using analytical and numerical methods is carried out by many researchers in the past to fill the knowledge gap. Some of the significant contributions are discussed here for the sake of brevity and to make the article self-standing.

Bending and the vibration behaviour of laminated composite plates are modeled by Cui et al. [3] using the FSDT mid-plane kinematics and solved using the discrete shear gap method. A new inverse hyperbolic shear deformation theory has been developed to analyse the static and buckling behaviour of laminated composite and sandwich plates by Grover et al. [4]. Hatami et al. [5] investigated the free vibration behaviour of moving laminated composite plate using exact and semi-analytical finite strip method in the framework of the CLPT. Huu-Tai and Seung-Eock [6] investigated the free vibration responses of laminated composite plate using two variable based on the refined plate theories. Jameel et al. [7] presented the buckling behaviour of laminated composite plate under the combined thermal and mechanical load. Jeng-Shian and Shyue [8] reported 3D elasticity solutions of thermal buckling responses of anti-symmetric angle-ply laminates using the higher-order displacement field. Kant and Swaminathan [9] presented analytical solutions of free vibration responses of laminated composite and sandwich plates using the higher order refined theory. Effect of the symmetric cross-ply laminations on the responses of laminated elastic plate is investigated by Khdeir and Librescu [10] using the HSDT mid-plane kinematics. Thermally induced buckling behaviour of laminated composite is analysed by Lee [11] using a layerwise theory in conjunction with finite element method (FEM). Matsunaga [12] reported the free vibration and the stability behaviour of angle-ply laminated composite and sandwich plate under the thermal loading and using power series expansion. Matsunaga [13] investigated the free vibration behaviour of cross-ply multi-layered composite plate in the framework of HSDT. Putcha and Reddy [14] reported stability and vibration responses of laminated composite plate using a mixed element based refined plate theory. Reddy and Liu [15] studied the static and free vibration behaviour of laminated elastic composite shells based on the HSDT kinematic model. Shukla and Nath [16] investigated the buckling and post-buckling strength of angleply laminated plates analytically under the thermo-mechanical loading in the framework of the FSDT by taking the geometrical non-linearity in von-Karman sense. Topal and Uzman [17] reported optimum design of laminated composite plate using four node Lagrangian finite element approach in the framework of the FSDT. Whitney and Pagano [18] investigated the free vibration behaviour of laminated plate using the FSDT kinematics. Xiang et al. [19] analysed the free vibration characteristics of laminated composite shells based on 
the FSDT in conjunction with meshless global collocation method. Free vibration behaviour of laminated composite plates is obtained by Xiang and Kang [20] using a meshless local collocation method based on thin plate spline radial basis function. Zhen and Wanji [21] investigated the buckling behaviour of angle-ply composite and sandwich plates. Thermomechanical buckling behaviour of laminated composite and sandwich plates are analysed by Zhen and Wanji [22] in the framework of the global-higher order theory. Thermal postbuckling strength of laminated cylindrical shells is investigated by Shen [23] using boundary layer theory. Thermal buckling responses of skew fibre-reinforced composite and sandwich plates are obtained by Kant and Babu [24] using shear deformable finite element model in the framework of the FSDT and the HSDT mid-plane kinematics. Mantari et al. [25] studied the static and dynamic behaviour of laminated composite and sandwich flat/shell panel based on a new higher-order shear deformation theory. Buckling and vibration behaviour of laminated composite plate/shell structure is investigated by Nguyen-Van et al. [26] using a smoothed quadrilateral flat shell element in the framework of the FSDT. Some of the recent investigations are completed on mechanical system design and optimisation using simulation tool, soft computing steps and available numerical methods under combined action of loading [27-31].

It is clear from the above review that, various attempts have already been made on the free vibration and the buckling behaviour of laminated structures under the combined thermomechanical load using the numerical approach. However analysis using commercial FEM tool of such structure are very limited in number whereas these tools have capability to solve such problems with less computational cost without affecting the accuracy of the final response. It is also worthy to mention that, the commercial packages are well appreciated by many industries their ease applicability, less effort and to achieve the real life situation without ignoring the complexities. Hence, the present investigation aims to analyse the thermomechanical buckling and the free vibration behaviour of shear deformable laminated composite flat/curved panels using thermo-elastic properties. The present finite element model is developed using ANSYS parametric design language (APDL) code in ANSYS environment. The critical buckling load parameter and fundamental frequencies have been obtained for different geometrical parameters and material properties for different support conditions using Block-Lanczo's method and validated by comparing the responses to that available numerical responses. In this analysis, the temperature distribution field is assumed to be uniform and linear through the panel thickness. The vibration and stability responses are computed by varying design parameters like thickness ratio $(a / h)$, modular ratio $\left(E_{1} / E_{2}\right)$, curvature ratio $(R / a)$, lamination scheme and support condition to highlight the effectiveness of the present proposed simulation model.

\section{Finite Element Modelling}

For the present analysis laminated composite model has been developed using APDL code in ANSYS. The general geometry and dimension of the laminated structure are same as in Fig. 1. It is well known that, the mathematical model in ANSYS is following the FSDT type mid-plane kinematics and conceded as:

$$
\begin{aligned}
u(x, y, z) & =u_{0}(x, y)+z \theta_{x}(x, y) \\
v(x, y, z) & =v_{0}(x, y)+z \theta_{y}(x, y) \\
w(x, y, z) & =w_{0}(x, y)+z \theta_{z}(x, y)
\end{aligned}
$$


Fig. 1 Laminated composite flat panel

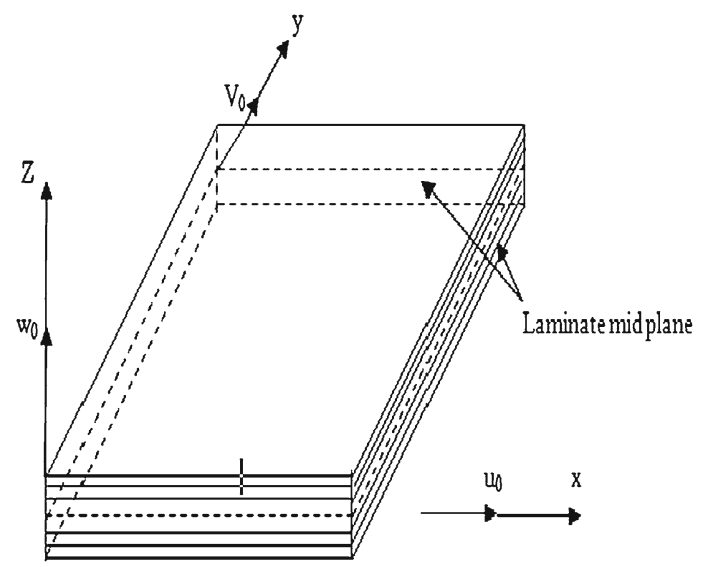

where, $u, v$ and $w$ denote the displacements of any point along the $(x, y, z)$ coordinates. $\mathrm{u}_{0}, \mathrm{v}_{0}$ and $\mathrm{w}_{0}$ are the in-plane and transverse displacements of a point $(x, y)$ on the midplane respectively and $\theta_{x}, \theta_{y}$ and $\theta_{z}$ are the rotations of normal to the mid-plane.

The following strain displacements are used to obtain the strains for any $k$ th lamina as follows:

$$
\{\varepsilon\}=\left\{\varepsilon_{x} \varepsilon_{y} \varepsilon_{z} \gamma_{z x} \gamma_{z y} \gamma_{x y}\right\}=\left\{u_{x} v_{y} w_{z} u_{z}+w_{x} v_{z}+w_{y} u_{y}+v_{x}\right\}^{T}
$$

where, $u_{x}=\partial u / \partial x, v_{y}=\partial v / \partial y$, and $w_{z}=\partial w / \partial z$ are the differential form of displacement terms with respect to the corresponding axis.

The generalized stress tensor of any $k$ th lamina via thermo-elastic constitutive relation is expressed as:

$$
\{\sigma\}^{k}=\left\{\sigma_{x} \sigma_{y} \sigma_{z} \tau_{y z} \tau_{x z} \tau_{x y}\right\}^{T}=[\bar{Q}]^{k}\left\{\varepsilon^{k}-\alpha^{k} \Delta T\right\}
$$

where, $\{\sigma\}^{k}$ is the stress tensor, $[\bar{Q}]^{k}$ transfer reduced stiffness matrix and $\alpha^{k}$ is the linear thermal expansion of the material. In addition to that, the $\Delta T$ is the temperature increment and it set to at zero for the free vibration analysis. The individual coefficients of transformed reduced stiffness matrix of any orthotropic material can be seen in [32].

Total strain energy of laminated flat/curved structure can be obtained due to the stresses and their strains i.e., using Eqs. (2) and (3) and the final form of the energy expression conceded as:

$$
U=\frac{1}{2} \int_{v}\{\varepsilon\}^{T}[\bar{Q}]\{\varepsilon\} d V
$$

The global displacement field of any point within the material continuum as in Eq. (1) can be expressed in terms of mid-plane displacements and corresponding thickness coordinate as:

$$
\{\bar{\delta}\}=\{u v w\}^{T}=[f]\{\delta\}
$$

where, $[f]$ is the function of thickness coordinate.

The kinetic energy of the vibrated laminated panel can be expressed as:

$$
T=\frac{1}{2} \int_{v}[\rho]\{\overline{\dot{\delta}}\}^{T}\{\overline{\dot{\delta}}\} d V
$$




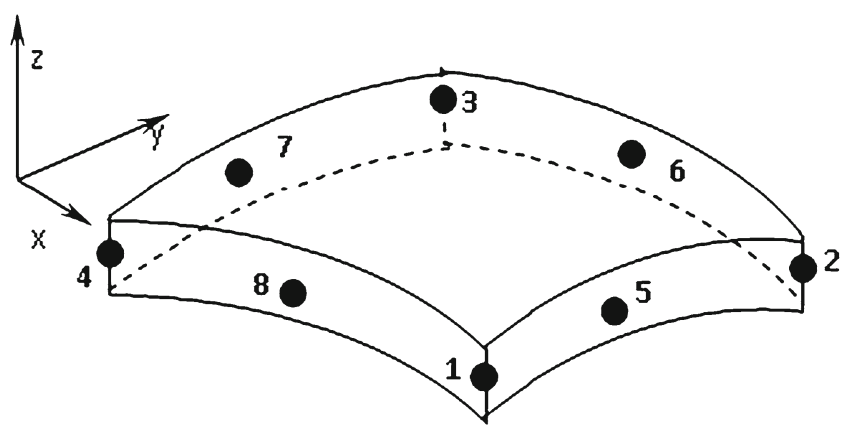

Fig. 2 A typical SHELL 281 element

where, $\rho$ is the density, $\{\overline{\dot{\delta}}\}$ and $\{\bar{\delta}\}$ are the velocity and the displacement vectors, respectively.

The external work done due to the temperature load will be obtained using the steps in [33]:

$$
W_{\Delta T}=\frac{1}{2} \int[\bar{\varepsilon}]\left\{N_{\Delta T}\right\} d V
$$

where, $\left[\bar{\varepsilon}_{G}\right]$ is the geometric strain vector and $\left\{N_{\Delta T}\right\}=\left\{N_{\Delta T x} N_{\Delta T y} N_{\Delta T x y}\right\}^{T}$ is the thermal load vector.

FEM has been proved to be a robust numerical technique [33] for the analysis of the layered structure with or without considering the environmental effect. In this analysis, an eight noded shell element (SHELL 281) is used from the ANSYS library [34] for the discretisation purpose. The element geometry can be seen in the Fig. 2 and it is capable of analysing thin to moderately thick shell/plate structures. This element has six degrees of freedom at each node called the translations and the rotations in the $x, y$, and $z$ axes. This element also includes the effect of transverse shear deformations to achieve the generality. The nodal displacements can be presented in the following form by considering the nodal shape functions and conceded as:

$$
\begin{aligned}
& u^{0}=\sum_{i=1}^{8} N_{i} u_{i}^{0}, v^{0}=\sum_{i=1}^{8} N_{i} v_{i}^{0}, w^{0}=\sum_{i=1}^{8} N_{i} w_{i}^{0}, \\
& \theta_{x}=\sum_{i=1}^{8} N_{i} \theta_{x i}, \theta_{y}=\sum_{i=1}^{8} N_{i} \theta_{y i}, \theta_{z}=\sum_{i=1}^{8} N_{i} \theta_{z i}
\end{aligned}
$$

Now, the Eq. (8) can be rewritten in a generalised form for any $i$ th node and expressed as:

$$
\begin{aligned}
& \left\{\delta_{i}^{*}\right\}=\left\{u_{i}^{0} v_{i}^{0} w_{i}^{0} \theta_{x i}^{0} \theta_{y i}^{0} \theta_{z i}^{0}\right\} \\
& \left\{\delta_{i}^{*}\right\}=\left[N_{i}\right]\left\{\delta_{i}\right\}
\end{aligned}
$$

where, $\left[N_{i}\right]$ nodal shape function of an eight noded element and the values can be seen in [33].

Substituting the value of nodal displacement in Eqs. (2), (4), (6) and (7) and it can be rewritten as: 


$$
\begin{aligned}
\{\varepsilon\} & =\left[B_{i}\right]\left\{\delta_{i}\right\} \\
T & =\frac{1}{2} \int_{A}[\rho][N]_{i}^{T}[N]_{i}\left\{\overline{\dot{\delta}}_{i}\right\} d V \\
U & =\frac{1}{2} \int\left[\mathrm{B}_{\mathrm{i}}\right]^{T}\left\{\delta_{i}\right\}^{T}[\mathrm{D}]\left[\mathrm{B}_{\mathrm{i}}\right]\left\{\delta_{i}\right\} d A-\{F\}_{\Delta T i}-\{F\}_{m i} \\
\{W\} & =\frac{1}{2} \int\left[\varepsilon_{G}\right]^{T}\left[\mathrm{D}_{G}\right]\left[\varepsilon_{G}\right] d A
\end{aligned}
$$

where, $\left[B_{i}\right],\{F\}_{\Delta T},\{F\}_{m}$ and $\left[\varepsilon_{G}\right]$ are the strain displacement relation matrix, thermal force vector, mechanical force vector and the geometric strain vectors, respectively.

The final form of the governing equation can be obtained by using Hamilton's principle as follows:

$$
\int_{0}^{t} L d t=0
$$

where, $L=T-(U+W)$.

Now, the Eq. (15) will be modified by using the Eqs. (11)-(14) and conceded to the following form:

$$
[\mathrm{M}]\{\ddot{\delta}\}+[\mathrm{K}]\{\delta\}=\{F\}_{\Delta T}-\{F\}_{m}
$$

where, $[\mathrm{M}]$ and $[\mathrm{K}]$ are the global mass and stiffness matrix.

The governing equation for free vibrated panel is obtained by dropping the force terms from the Eq. (16) and turned to the eigenvalue type of equation as follows:

$$
[\mathrm{M}]\{\ddot{\delta}\}+[\mathrm{K}]\{\delta\}=0
$$

In the similar fashion, the governing equation of buckling for the laminated panel can also be obtained by dropping the inertia force term from Eq. (16) and conceded as:

$$
[\mathrm{K}]\{\delta\}=\{F\}_{\Delta T}-\{F\}_{m}
$$

Now, to obtain the eigenvalue type of buckling equation obtained by dropping the force vectors from Eq. (18) and considering their effect in terms of geometrical matrix as:

$$
\left\{[\mathrm{K}]+\lambda_{c r}\left[\mathrm{~K}_{G}\right]\right\}\{\delta\}=0
$$

where, $\left[\mathrm{K}_{G}\right]$ is the geometric stiffness matrix and $\lambda_{c r}$ is the critical thermal/mechanical load at which the structure buckles. The evaluation steps of geometric stiffness matrix $\left[\mathrm{K}_{G}\right]$ can be seen in [33].

\section{Results and Discussions}

The free vibration and buckling behaviour of laminated composite curved/flat panel are computed using the simulation model developed in ANSYS through APDL code. The validity and comprehensive behaviour of the present finite element model have been checked by comparing the responses of to that available published results. The effects of different parameters such as the modular ratios, the thickness ratios, the curvature ratios, support conditions and number of layers on the natural frequencies and critical buckling loads of the laminated composite panel is highlighted through solving numerous examples. In this study, all the 
Fig. 3 A full square flat panel with meshes of $\mathrm{m} \times \mathrm{m}$

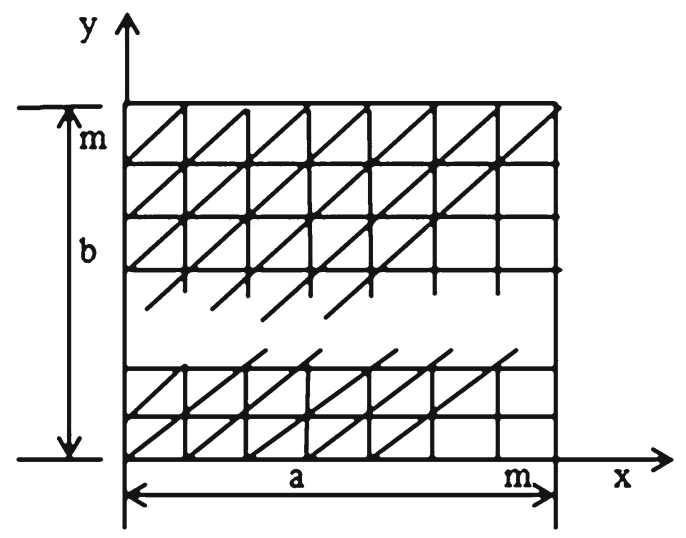

layers are assumed to have the uniform thickness, the thermal expansion coefficients and elastic constants are assumed to be independent of temperature. In addition to this, a uniform temperature has been considered for throughout the analysis through the thickness of the laminated panel. The mesh configuration for any general finite element analysis through its can be seen in Fig. 3.

The following lamina properties are used for the vibration and the buckling analysis:

M1: $E_{1} / E_{2}=$ open; $G_{12}=G_{13}=0.6 E_{2} ; G_{23}=0.5 E_{2} ; v_{12}=0.25 ; \rho=1$

M2: $E_{1} / E_{2}=$ open; $G_{12}=G_{13}=0.6 E_{2} ; G_{23}=0.5 E_{2} ; v_{12}=v_{13}=0.21 ; v_{23}=0.49$

M3: $E_{1} / E_{2}=$ open; $G_{12}=G_{13}=0.65 E_{2} ; G_{23}=0.639 E_{2} ; v_{12}=v_{13}=0.21$; $v_{23}=0.33 ; \alpha_{12}=-0.21 \alpha_{0}, \alpha_{23}=16.0 \alpha_{0}$ and $\alpha_{0}=1 \times 10^{-6} /{ }^{\circ} \mathrm{C}$

M4: $E_{1} / E_{2}=$ open; $G_{12}=G_{13}=0.5 E_{2} ; G_{23}=0.3356 E_{2} ; v_{12}=v_{13}=0.3$; $v_{23}=0.49 ; \alpha_{12}=0.015 \alpha_{0}, \alpha_{23}=\alpha_{13}=16.0 \alpha_{0}$ and $\alpha_{0}=1 \times 10^{-6} /{ }^{\circ} \mathrm{C}$

The following sets of boundary conditions are used for the present analysis.

(a) Simply supported condition (S):

$$
\begin{aligned}
v_{0}, w_{0}, \theta_{x}, \theta_{z}=0 & \text { at } \mathrm{x}=0, \mathrm{a} \\
u_{0}, w_{0}, \theta_{y}, \theta_{z}=0 & \text { at } \mathrm{y}=0, \mathrm{~b}
\end{aligned}
$$

(b) Clamped condition (C):

$$
u_{0}, v_{0}, w_{0}, \theta_{x}, \theta_{y}, \theta_{z}=0 \text { at } \mathrm{x}=0 \text {, a and } \mathrm{y}=0, \mathrm{~b}
$$

The following forms of non-dimensional parameters are used in the whole analysis If not stated otherwise.

Frequency: $\bar{\omega}=\left(\omega a^{2} / h\right)\left(\sqrt{\rho / E_{2}}\right)$

Critical buckling load: $\bar{N}_{x x}=\lambda a^{2} / E_{2} h^{3}$

Critical buckling temperature: $\Delta T_{c r}^{*}=\Delta T_{c r} \times \alpha_{0} \times 1000$

\section{Convergence and Comparison Study}

In this section, the convergence behaviour of the present finite element simulation model have been checked and compared with those available published results. For the validation purpose, all problems have been solved by setting the geometrical and material properties same as to the references. 
Table 1 Convergence and comparison behaviour of non-dimensional fundamental frequency parameter of square simply supported laminated composite flat panel $\left(E_{1} / E_{2}=40\right)$

\begin{tabular}{lllllll}
\hline $\begin{array}{l}\text { No. of layers } \\
\text { Mesh size }\end{array}$ & $\begin{array}{l}\left(0^{\circ} / 90^{\circ}\right)_{2} \\
\mathrm{a} / \mathrm{h}\end{array}$ & \multicolumn{3}{c}{$\left(0^{\circ} / 90^{\circ}\right)_{3}$} & & \multicolumn{2}{c}{$\left(0^{\circ} / 90^{\circ}\right)_{5}$} \\
\cline { 2 - 6 } & 10 & 50 & 10 & 50 & 10 & 50 \\
\hline Present $(6 \times 6)$ & 14.4224 & 17.1606 & 15.2499 & 18.0541 & 15.674 & 18.4961 \\
Present $(7 \times 7)$ & 14.4212 & 17.1591 & 15.2493 & 18.0525 & 15.6734 & 18.4942 \\
Present $(8 \times 8)$ & 14.4205 & 17.1581 & 15.2487 & 18.0516 & 15.6728 & 18.4936 \\
Present $(9 \times 9)$ & 14.4205 & 17.1578 & 15.248 & 18.0513 & 15.6728 & 18.493 \\
Present $(10 \times 10)$ & 14.4199 & 17.1578 & 15.248 & 18.051 & 15.6728 & 18.493 \\
FSDT [18] & 14.9214 & 17.1899 & 15.501 & 18.0673 & 15.779 & 18.4995 \\
TSDT [2] & 14.8463 & 17.1849 & 15.4632 & 18.0644 & 15.77 & 18.4984 \\
RPT [6] & 14.8463 & 17.1849 & 15.4632 & 18.0644 & 15.77 & 18.4984 \\
\hline
\end{tabular}

\section{Validation and Convergence of Free Vibration}

In this example, the free vibration responses of square simply supported laminated composite flat panel is computed for two thickness ratios $(a / h=10$ and 50) and three anti-symmetric cross-ply laminations $\left[\left(0^{\circ} / 90^{\circ}\right)_{2},\left(0^{\circ} / 90^{\circ}\right)_{3}\right.$ and $\left.\left(0^{\circ} / 90^{\circ}\right)_{5}\right]$ by using M1 material properties with $E_{1} / E_{2}=40$ as in the reference. The results are compared with those available published article and presented in Table 1 . It is worthy to mention that, the reference results are computed using three different established theories (third-order shear deformation theory, TSDT, refined plate theory, RPT and the FSDT) whereas the present results are computed using the proposed simulation model. The comparison study clearly indicates that the present model shows very good agreement with those established theories without hampering the accuracy of the result. However, it is also interesting to note that the differences between the results are below 1 and $3.5 \%$ for the thin and thick panels respectively. It is also understood that the present model is capable to analysing the free vibration behaviour of any layered structure with sufficient accuracy and less computational cost. Based on the convergence study, a $(10 \times 10)$ mesh is used to obtain the free vibration responses for further analysis.

\section{Validation and Convergence of Mechanical Buckling}

Now the present model has been extended to validate the mechanical buckling load parameter of square simply supported cross-ply $\left(0^{\circ} / 90^{\circ} / 0^{\circ}\right)$ laminated composite flat panel for two different modular ratio $\left(E_{1} / E_{2}=2\right.$ and 20) using M2 type material properties and $a / h=10$. The non-dimensional buckling load computed using the present model and the reference values are presented in Table 2. In addition to that the convergence behaviour of the present simulation model also presented in the table for different mesh division. The results clearly indicate that, the present model is showing good convergence rate and the differences are within the expected line. It is also important to mention that the differences between the results are well below i.e., within $3.5 \%$ with the HSDT model. The mechanical buckling responses of laminated composite panel is computed using a $(14 \times 14)$ mesh further based on the convergence. 
Table 2 Convergence and comparison behaviour of the non-dimensional buckling load parameter of square laminated simply supported composite flat panel $\left(0^{\circ} / 90^{\circ} / 0^{\circ}\right)(a / h=10)$

\begin{tabular}{lll}
\hline Mesh density & $E_{1} / E_{2}=2$ & $E_{1} / E_{2}=20$ \\
\hline $10 \times 10$ & 3.3432 & 7.9723 \\
$11 \times 11$ & 3.0414 & 7.2474 \\
$12 \times 12$ & 2.7895 & 6.6434 \\
$13 \times 13$ & 2.5761 & 6.1324 \\
$14 \times 14$ & 2.393 & 5.6944 \\
HSDT [22] & 2.364 & 5.516 \\
\hline
\end{tabular}

Table 3 Convergence and comparison study of non-dimensional buckling temperature for square simply supported laminated composite flat panel $\left( \pm 45^{\circ}\right)_{3}(a / h=10)$

\begin{tabular}{llll}
\hline Mesh density & $E_{1} / E_{2}=1$ & $E_{1} / E_{2}=2$ & $E_{1} / E_{2}=3$ \\
\hline $16 \times 16$ & 1.6678 & 2.4695 & 3.2414 \\
$17 \times 17$ & 1.6678 & 2.4696 & 3.2416 \\
$18 \times 18$ & 1.668 & 2.4697 & 3.2417 \\
$19 \times 19$ & 1.668 & 2.4698 & 3.2418 \\
$20 \times 20$ & 1.668 & 2.4698 & 3.2419 \\
HSDPT [14] & 1.6685 & 2.4721 & 3.249 \\
FSDPT [14] & 1.6685 & 2.4723 & 3.25 \\
\hline
\end{tabular}

Validation and Convergence of Thermal Buckling

In the continuation the above two cases, one more example has been solved using the present simulation model for thermal buckling behaviour. In this example, the convergence and comparison behaviour of simply supported square anti-symmetric angle-ply $\left[ \pm 45^{\circ}\right]_{3}$ laminated composite flat panel is analysed for three different modular ratios $\left(E_{1} / E_{2}=1,2,3\right)$ with M3 material properties and the responses are tabulated in Table 3 by setting $a / h=10$. The results clearly indicate that the present model not only converging well but also showing excellent agreement with the available published literature. However, the reference results are solved using both higher-order shear deformation plate theory (HSDPT) and the first-order shear deformation plate theory (FSDPT) the differences are so small that it can be neglected. Based on the convergence, a $(20 \times 20)$ mesh is used to compute the responses further for the thermal buckling responses throughout the analysis.

Based on the each convergence and the comparison studies on the free vibration and the thermo-mechanical buckling of laminated composite panel structure, the present simulation model is further extended to analyse the effectiveness through solving wide variety of numerical examples for different parameters in the following section.

\section{Parametric Study of Free Vibration}

In this section, the fundamental frequency responses of laminated composite flat panel are analysed for two different support conditions (CCCC and CSCS). The non-dimensional fundamental frequency responses are computed using the APDL code and presented in Figs. 4 and 5, respectively. The responses are examined for five different thickness ratios $\left(a / h=5,10,15,20\right.$, and 50), five modular ratios $\left(E_{1} / E_{2}=10,20,30,40\right.$ and 50) and number of layups $\left[\left(0^{\circ} / 90^{\circ}\right)_{2},\left(0^{\circ} / 90^{\circ}\right)_{3},\left(0^{\circ} / 90^{\circ}\right)_{5}\right]$ by setting the composite properties as M1. It is clear from the figures that the non-dimensional fundamental frequency 


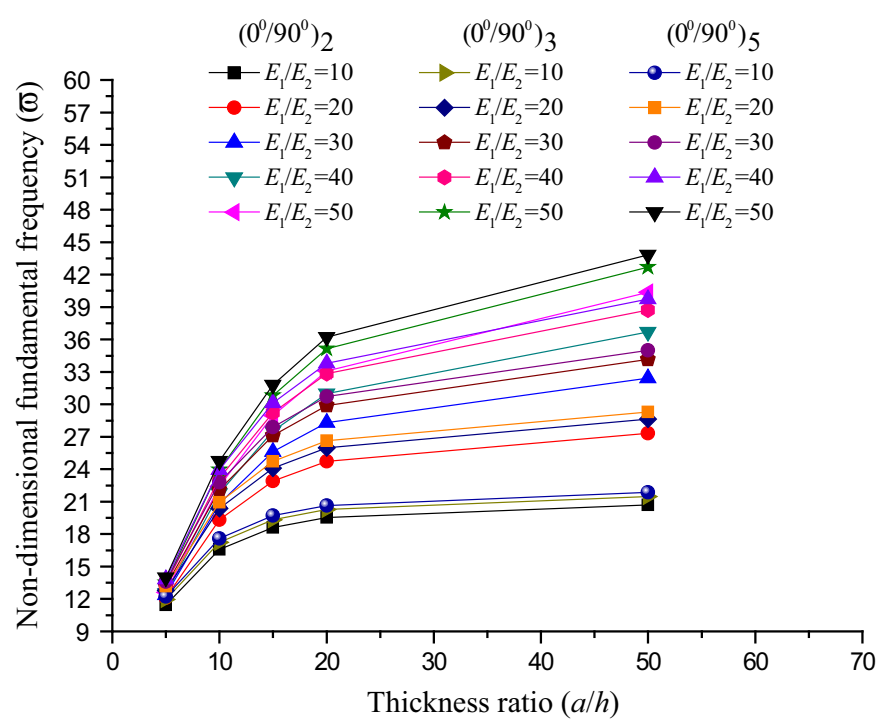

Fig. 4 Non-dimensional fundamental frequency of laminated composite flat panel for all edges clamped (CCCC) condition

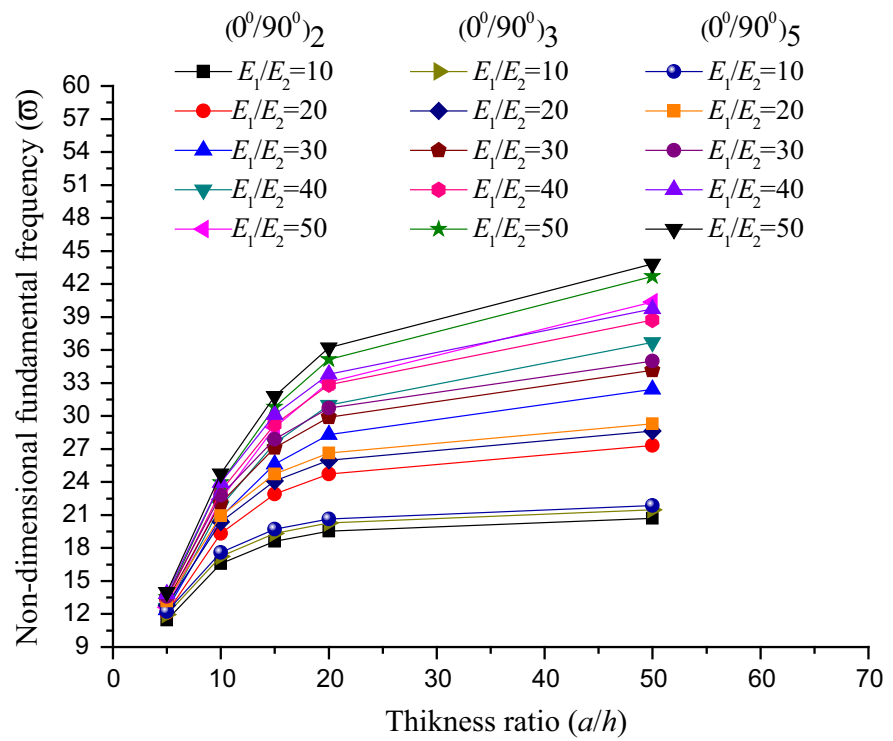

Fig. 5 Non-dimensional fundamental frequency of laminated composite flat panel for two edges clamped and two edges simply supported (CSCS) condition

increases with increasing of all the parameters i.e., the thickness ratio, the modular ratio and the number of layers. It is because of the fact that as the thickness ratio increases the panel becomes thin and the non-dimensional frequency goes up. Similarly, the structural stiffness of the layered structure directly depend upon on the number of layers and modular ratio and the responses are following the expected line for each of the parameters. 
Table 4 Non-dimensional fundamental frequency responses of laminated composite cylindrical and spherical panel $(R / a=10)$

\begin{tabular}{llllll}
\hline Panel geometry & $\begin{array}{l}\text { Lamination } \\
\text { scheme }\end{array}$ & $\begin{array}{l}\text { Support } \\
\text { condition }\end{array}$ & \multicolumn{2}{l}{$a / h$} & \\
\cline { 5 - 6 } Cylindrical & & & 10 & 20 & 100 \\
& $\left(0^{\circ} / 90^{\circ}\right)_{5}$ & SSSS & 12.1919 & 14.1736 & 16.4682 \\
& & CCCC & 18.1402 & 26.7965 & 44.2726 \\
& $\left( \pm 45^{\circ}\right)_{5}$ & SSSS & 11.7847 & 15.4164 & 25.2584 \\
& & CCCC & 15.2449 & 21.9824 & 36.2364 \\
\hline Spherical & & & 5 & 10 & 100 \\
& $\left(0^{\circ} / 90^{\circ}\right)_{S}$ & SSSS & 9.7848 & 13.09353 & 21.3163 \\
& & CCCC & 12.5833 & 20.9003 & 54.1315 \\
& $\left( \pm 45^{\circ}\right)_{S}$ & SSSS & 10.1916 & 14.3539 & 40.2174 \\
& & CCCC & 12.4064 & 19.6010 & 50.3867 \\
\hline
\end{tabular}

To show the generality of the present developed model, it is extended to analyse for two more geometries say, cylindrical and spherical laminated shell panels of the square base. The responses are computed for different thickness ratios, lamination schemes (cross-ply and angle-ply) and support conditions (SSSS and CCCC) using the M1 type material properties by setting $E_{1} / E_{2}=25$. The non-dimensional fundamental frequency responses are presented Table 4. It is clear from the results that, the fundamental frequency responses are higher for clamped support as compared to simply supported case. It is also interesting to note that the cylindrical panel is showing higher non-dimensional frequencies for lower thickness ratios i.e., $a / h=10$ and 20 and the responses are revert for $a / h=100$. In addition, it is also observed that the lamination scheme affect the frequency behaviour of the layered structures considerably.

Parametric Study of Mechanical Buckling

It is well known that the buckling load greatly depend on two major parameters such as the support condition and the type of loading. In this section, two different examples of mechanical buckling are analysed under the influence of biaxial and uniaxial compressive load and presented in Fig. 6a, b, respectively. The buckling responses are computed for square cross-ply laminated composite flat panel $\left(0^{\circ} / 90^{\circ} / 0^{\circ} / 90^{\circ} / 0^{\circ}\right)$ for six thickness ratios $\left(a / h=5,10,15,20,50\right.$ and 100) and five modular ratios $\left(E_{1} / E_{2}=1,2,3,4\right.$ and 5) using the M2 composite properties with CCSS support. It is clear from the Fig. 6 that, the non-dimensional buckling load parameter is showing decreasing type of behaviour as the thickness ratio increases whereas a reverse trend is observed for the orthotropicity ratios.

Further, two more examples are solved for two different geometries i.e., the cylindrical and the spherical laminated composite shell panel under uniaxial loading with simply support (SSSS) condition. The buckling responses are obtained for three thickness ratios $(a / h=2$, 20 , and 100), four curvature ratios $(R / a=5,10,15$, and 20) and two lamination schemes $\left[\left(0^{\circ} / 90^{\circ} / 0^{\circ}\right)\right.$ and $\left.\left(0^{\circ} / 90^{\circ} / 0^{\circ} / 90^{\circ} / 0^{\circ}\right)\right]$ with the same material properties as in the earlier example. The non-dimensional buckling load parameters of cylindrical and spherical shell 

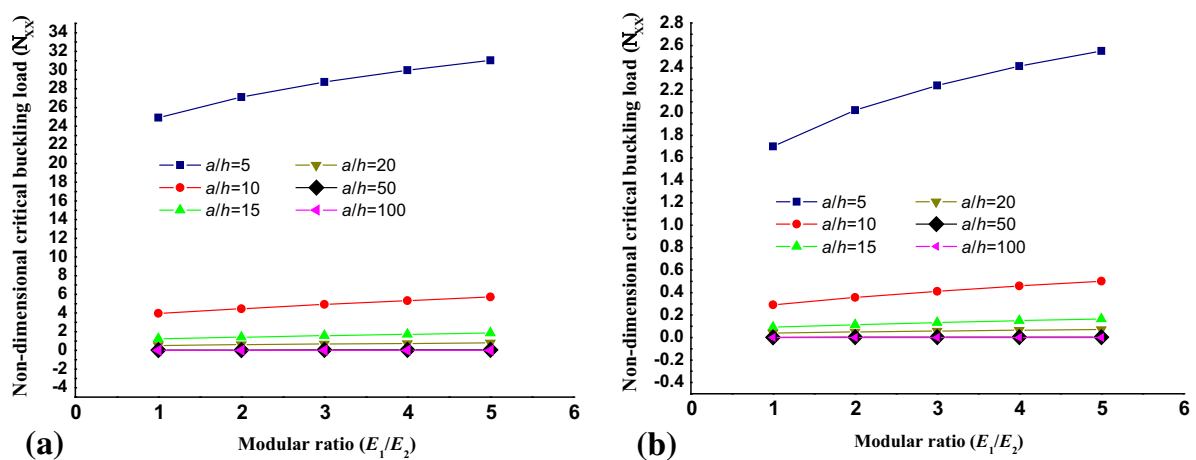

Fig. 6 Non-dimensional critical buckling load responses of laminated composite flat panel $\left(0^{\circ} / 90^{\circ} / 0^{\circ} / 90^{\circ} / 0^{\circ}\right)$ with CCSS support. a Biaxial loading. b Uniaxial loading
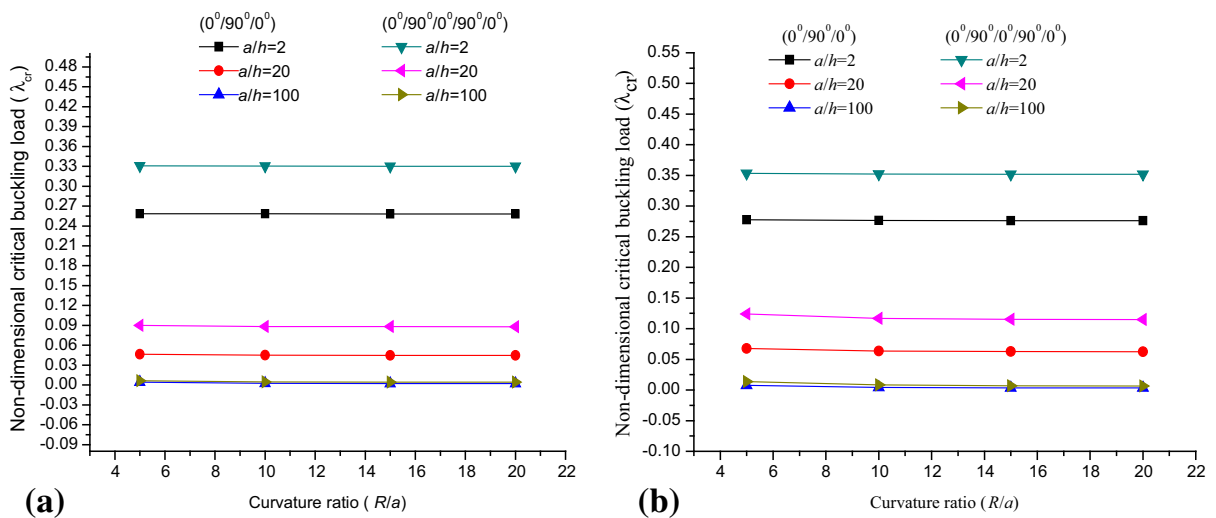

Fig. 7 Non-dimensional critical buckling load responses of laminated composite panel $\left(\lambda_{c r}=N_{X} / E_{2} h\right)$. a Cylindrical; b spherical

panels are presented in Fig. 7a, b, respectively. It is clear from figures that the buckling load parameters are decreasing with an increase in the curvature ratios and the thickness ratios and this is because the subsequent reduction in panel stiffness.

\section{Parametric Study of Thermal Buckling}

The thermal buckling strength of anti-symmetric angle-ply laminated composite flat panel is computed for five thickness ratios $(a / h=5,10,15,20,50$ and 100), five modular ratios $\left(E_{1} / E_{2}=1,2,3,4\right.$ and 5), two stacking sequences $\left[\left( \pm 15^{\circ}\right)_{3}\right.$ and $\left.\left( \pm 45^{\circ}\right)_{3}\right]$ and two support conditions (CCCC and CSCS). The responses are computed using the composite properties as M3 and presented in Figs. 8a, b and 9a, b for CCCC and CSCS supports, respectively. It is clear from all the four figures presented in Figs. 8 and 9, the non-dimensional buckling temperature increase and decrease, even though, both the modular ratios and the thickness ratios increase. This can also be noted that the non-dimensional buckling temperature of the flat angle panel ply panel increases as the ply angle increases for each case are analysed in this present example. 


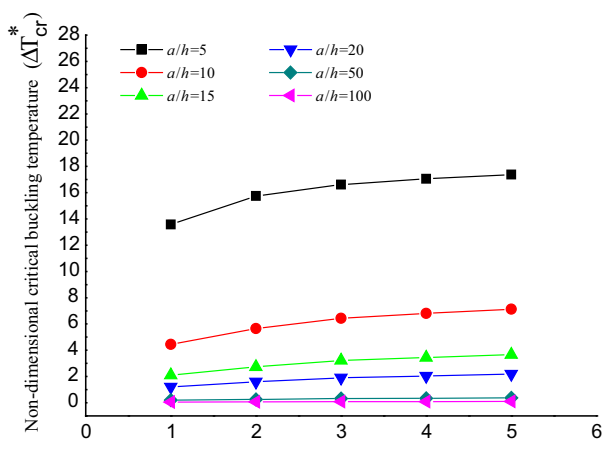

(a)

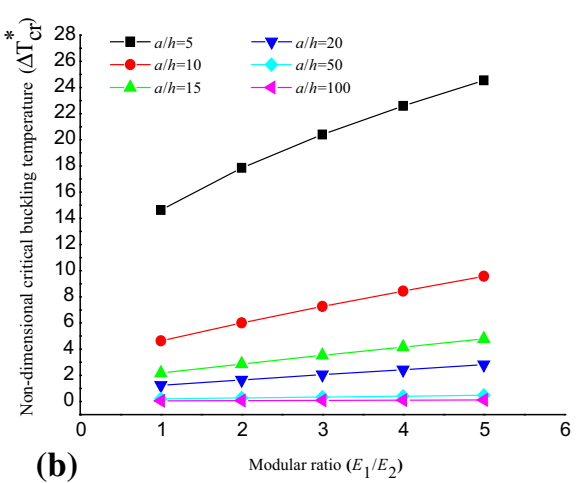

(b)

Fig. 8 Non-dimensional critical buckling temperature of clamped laminated composite flat panel. $\mathbf{a}\left( \pm 15^{\circ}\right)_{3}$; b $\left( \pm 45^{\circ}\right)_{3}$

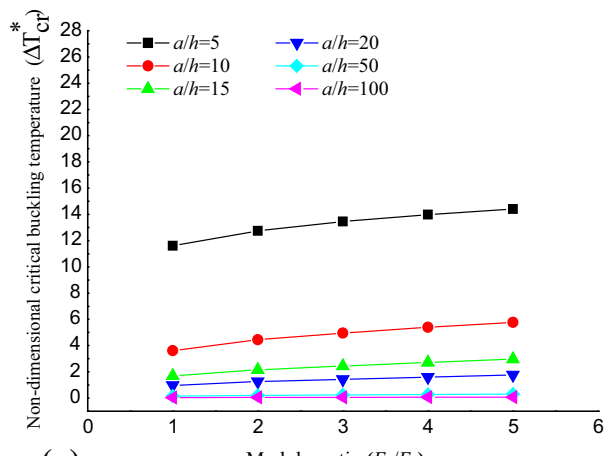

(a)

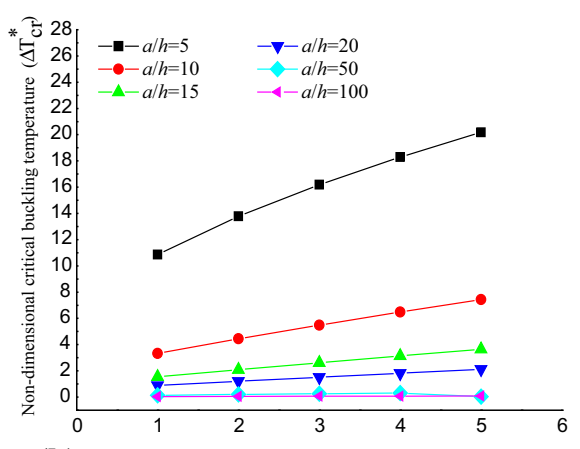

(b)

Fig. 9 Non-dimensional critical buckling temperature of laminated composite flat panel with CSCS support. a $\left( \pm 15^{\circ}\right)_{3} ; \mathbf{b}\left( \pm 45^{\circ}\right)_{3}$

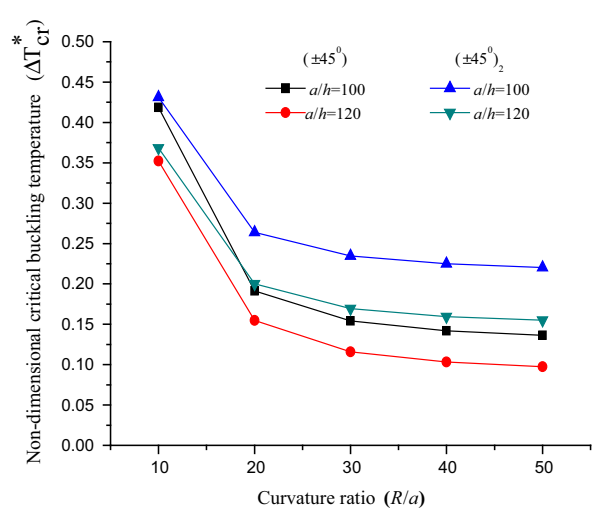

(a)

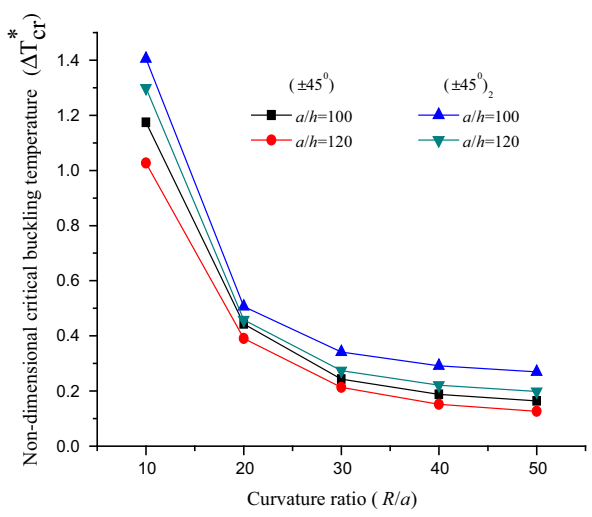

(b)

Fig. 10 Non-dimensional critical buckling temperature of laminated composite panel for CCCC condition. a Cylindrical; b spherical 
Further, two more problems on thermal buckling behaviour of clamped composite panel have been analysed for two different geometries i.e., the cylindrical and the spherical shell panel. The responses are computed for five curvature ratios $(R / a=10,20,30,40$ and 50), two thickness ratios $(a / h=100$ and 120$)$ and two lamination scheme $\left(\left[ \pm 45^{\circ}\right]\right.$ and $\left.\left[ \pm 45^{\circ}\right]_{2}\right)$ using M3 and M4 type material properties and presented in Fig. 10a, b, respectively. It is observed from the figures that, the non-dimensional buckling temperatures decrease when both the curvature ratios and the thickness ratios increase. This is because of the fact that as the thickness ratio increases the structure becomes thin and the structure follows the expected line. It is also noted that the non-dimensional buckling temperature is showing increasing trend as the number of layers increases, it is because the stiffness increases as the number of layers increases.

\section{Conclusions}

In this article, a finite element model of laminated composite flat/curved panel is developed in ANSYS simulation package using APDL code to analyse the free vibration and buckling behaviour of the layered structures numerically. The comprehensive behaviour of the developed simulation model has been checked and it clearly indicates that the present simulation model not only capable to solve the mechanical responses easily but also reduced the computational cost considerably. The comparison study also indicated the excellent accuracy for all the different classes of problem analysed using the present simulation model with those available published literature. The present free vibration and the thermo-mechanical buckling behaviour of laminated composite flat/curved panels has been analysed for different parameters such as the thickness ratios, the lamination schemes, the modular ratios and support condition. Based on the computation of variety numerical example the following conclusions are drawn:

- In the free vibration analysis, the non-dimensional fundamental frequencies increase as the thickness ratios, the modular ratios and the number of plies increases. The nondimensional fundamental frequencies are showing higher for clamped support.

- The of critical buckling load parameters of laminated composite flat/curved panel under the mechanical load decreases as the thickness ratios increase. However, the load parameter increases as the modular ratios and the number of plies increases.

- In the thermal buckling case, the critical buckling temperature parameter decreases as the thickness ratios increase and it increases as the modular ratios and the number of plies increases. The clamped panel is showing higher thermal buckling strength as compared to other support.

- It is also observed that, the spherical panels are showing higher buckling (thermal and mechanical) strength in comparison to cylindrical and flat panel. But the cylindrical panels are showing higher fundamental frequencies in comparison to spherical and flat panel except for thin laminates.

\section{References}

1. http://www.jyi.org/issue/the-boeing-787-dreamliner-designing-an-aircraft-for-the-future

2. Reddy, J.N.: Mechanics of Laminated Composite Plate: Theory and Analysis. CRC Press, Boca Raton (2004) 
3. Cui, X.Y., Liu, G.R., Li, G.Y.: Bending and vibration responses of laminated composite plates using an edge-based smoothing technique. Eng. Anal. Bound. Elem. 35, 818-826 (2011)

4. Grover, N., Maiti, D.K., Singh, B.N.: A new inverse hyperbolic shear deformation theory for static and buckling analysis of laminated composite and sandwich plates. Compos. Struct. 95, 667-675 (2013)

5. Hatami, S., Azhari, M., Saadatpour, M.M.: Free vibration of moving laminated composite plates. Compos. Struct. 80, 609-620 (2007)

6. Huu-Tai, T., Seung-Eock, K.: Free vibration of laminated composite plates using two variable refined plate theory. Int. J. Mech. Sci. 52, 626-633 (2010)

7. Jameel, A.N., Ibtehal, A.S., Hasanain, I.N.: Buckling analysis of composite plates under thermal and mechanical loading. J. Eng. 18, 1365-1390 (2012)

8. Jeng-Shian, C., Shyue-Y, L.: Thermal buckling analysis of antisymmetric angle-ply laminates based on a higher-order displacement field. Compos. Sci. Technol. 41, 109-128 (1991)

9. Kant, T., Swaminathan, K.: Analytical solution for free vibration of laminated composite and sandwich plates based on a higher refined theory. Compos. Struct. 53, 75-85 (2001)

10. Khdeir, A.A., Librescu, L.: Analysis of symmetric cross-ply laminated elastic plates using a higher-order theory Part II-buckling and free vibration. Compos. Struct. 9, 259-277 (1988)

11. Lee, J.: Thermally-induced buckling of laminated composites by a layerwise theory. Comput. Struct. $\mathbf{6 5}$, 917-922 (1997)

12. Matsunaga, H.: Free vibration and stability of angle-ply laminated composite and sandwich plates under thermal loading. Compos. Struct. 77, 249-262 (2007)

13. Matsunaga, H.: Vibration of cross-ply laminated composite plates subjected to initial in-plane stresses. Thin-Walled Struct. 40, 557-571 (2002)

14. Putcha, N.S., Reddy, J.N.: Stability and natural vibration analysis of laminated plates by using a mixed element based on a refined plate theory. J. Sound Vib. 104, 285-300 (1986)

15. Reddy, J.N., Liu, C.F.: A higher-order shear deformation theory of laminated elastic shells. Int. J. Eng. Sci. 23, 319-330 (1985)

16. Shukla, K.K., Nath, Y.: Analytical solution for buckling and post-buckling of angle-ply laminated plates under thermo-mechanical loading. Int. J. Non-Linear Mech. 36, 1097-1108 (2001)

17. Topal, U., Uzman, U.: Optimum design of laminated composite plates to maximize buckling load using MFD method. Thin-Walled Struct. 45, 660-669 (2007)

18. Whitney, J.M., Pagano, N.J.: Shear deformation in heterogeneous anisotropic plates. J. Appl. Mech.-T ASME 37, 1031-1036 (1970)

19. Xiang, S., Bi, Z., Jiang, S., Jin, Y., Yang, M.: Thin plate spline radial basis function for the free vibration analysis of laminated composite shells. Compos. Struct. 93, 611-615 (2011)

20. Xiang, S., Kang, G.: Local thin plate spline collocation for free vibration analysis of laminated composite plates. Eur. J. Mech. A/Solids 33, 24-30 (2012)

21. Zhen, W., Wanji, C.: Buckling analysis of angle-ply composite and sandwich plates by combination of geometric stiffness matrix. Comput. Mech. 39, 839-848 (2007)

22. Zhen, W., Wanji, C.: Thermomechanical buckling of laminated composite and sandwich plates using global-local higher order theory. Int. J. Mech. Sci. 49, 712-721 (2007)

23. Shen, H.S.: Thermal post-buckling analysis of imperfect stiffened laminated cylindrical shells. Int. J. Nonlinear Mech 32, 259-275 (1997)

24. Kant, T., Babu, C.S.: Thermal buckling analysis of skew fibre-reinforced composite and sandwich plates using shear deformable finite element models. Compos. Struct. 49, 77-85 (2000)

25. Mantari, J.L., Oktem, A.S., Guedes, Soares C.: Static and dynamic analysis of laminated composite and sandwich plates and shells by using a new higher-order shear deformation theory. Compos. Struct. 94, $37-49(2011)$

26. Nguyen-Van, H., Mai-Duy, N., Karunasena, W., Tran-Cong, T.: Buckling and vibration analysis of laminated composite plate/shell structures via a smoothed quadrilateral flat shell element with in-plane rotations. Comput. Struct. 89, 612-625 (2011)

27. Hatami, M., Ganji, D.D., Gorji-Bandpy, M.: Experimental and thermodynamical analyses of the diesel exhaust vortex generator heat exchanger for optimizing its operating condition. Appl. Therm. Eng. 75, 580-591 (2015)

28. Dehghana, M., Rahmani, Y., Ganji, D.D., Saedodin, S., Valipoura, M.S., Rashidi, S.: Convection-radiation heat transfer in solar heat exchangers filled with a porous medium: Homotopy perturbation method versus numerical analysis. Renew. Energy 74, 448-455 (2015)

29. Hatami, M., Jafaryar, M., Ganji, D.D., Gorji-Bandpy, M.: Optimization of finned-tube heat exchangers for diesel exhaust waste heat recovery using CFD and CCD techniques. Int. Commun. Heat Mass 57, 254-263 (2014) 
30. Sheikholeslami, M., Gorji-Bandpy, M., Ganji, D.D.: MHD free convection in an eccentric semi-annulus filled with nanofluid. J. Taiwan Inst. Chem. E 45, 1204-1216 (2014)

31. Mondal, S.P., Roy, T.K.: first order homogeneous ordinary differential equation with initial value as triangular intuitionistic fuzzy. J. Uncertain. Math. Sci 1-17 (2014). DOI:10.5899/2014/jums-00003

32. Jones, R.M.: Mechanics of Composite Materials, 2nd edn. Taylor and Francis Inc., London (1999)

33. Cook, R.D., Malkus, D.S., Plesha, M.E., Witt, R.J.: Concepts and Applications of Finite Element Analysis. Wiley, Singapore (2000)

34. ANSYS Inc., (2010), ANSYS 13.0 reference manual 